\title{
A Pesquisa Qualitativa em PSICOLOGIA CLÍNICA ${ }^{1}$
}

\author{
Elizabeth Batista Pinto
}

Instituto de Psicologia - USP

\begin{abstract}
Este artigo discute a metodologia qualitativa de pesquisa científica em psicologia clínica, considerando a ciência como uma construção da subjetividade humana e buscando compreender suas particularidades. Tal metodologia implica em um processo dinâmico de investigação dentro de um determinado sistema teórico. Assim a pesquisa qualitativa é sempre uma pesquisa-ação, pois conforme a ação vai sendo construída é também investigada e interpretada, modificando o próprio processo.
\end{abstract}

Descritores: Metodologia. Pesquisa qualitativa. Psicologia clínica. Ciência. Psicologia.

\section{A Pesquisa Qualitativa}

\begin{abstract}
ciência é constituída por teorias que resumem e organizam, de forma racional e coerente, muitas relações colocadas em evidência empiricamente. Assim, a ciência produz zonas de sentido da realidade do fenômeno estudado (Rey,1998) e se constitui a partir de paradigmas e modelos que fornessem problemas e soluções para um grupo de pesquisadores por um determinado período.
\end{abstract}

1 Este texto se articula ao conteúdo da disciplina "Metodologia Científica em Psicologia Clínica", ministrada pela autora em 1999, no programa de pós-graduação em Psicologia Clínica do Instituto de Psicologia - USP. Neste contexto, o professor Luiz Carlos Nogueira foi convidado a dar uma aula sobre "Pesquisa em Psicanálise", cuja transcrição e edição constitui o próximo artigo deste número da revista.

Psicologia USP, 2004, 15(1/2), 71-80 
Um paradigma é um conjunto de conceitos, valores, percepções e práticas aceito e compartilhado por uma comunidade científica a partir de uma determinada concepção da realidade e estruturado tendo por base um certo tipo de pensamento (Kuhn, 1972). Assim, um paradigma constitui-se por descobertas científicas universalmente reconhecidas que, por algum tempo, fornecem tanto questões como possíveis soluções a um grupo de pesquisadores.

O paradigma é, portanto, um modelo mental de referência, uma forma de ver os fenômenos que inclui crenças a partir das quais são filtradas percepções e conteúdos para a interpretação do mundo. Assim, quando adotamos um paradigma, além dos aspectos cognitivos do conhecimento incluem-se também os aspectos subjetivos, emocionais e as identificações, implicando na aceitação ou na rejeição das idéias de outros paradigmas concorrentes ou oponentes.

A evolução da ciência é paradigmática. Isto significa que, ao longo da história, a ciência apresenta ciclos paradigmáticos, cada um comprendendo uma fase inicial na qual podem se defrontar, na mesma especialidade, diferentes teorias. Dessa forma, a ciência progride tanto pela confirmação sistemática das hipóteses consideradas nas pesquisas, como também por sua refutação, sofrendo a influência de dados contraditórios, inconciliáveis ou inexplicáveis e das insatisfações com as teorias atuais.

A constante inquietude da ciência conduz frequentemente à substituição de uma teoria por outra mais englobante e à reinterpretação das teorias por novos dados e novas questões (Kuhn, 1972). A pesquisa científica busca descobrir regularidades que ampliem os conhecimentos no campo específico e possibilitem o progresso da ciência. Tal avanço, em geral, ocorre de forma interativa, por um processo cumulativo gradual de aproximações sucessivas ao problema estudado, no qual cada solução é função do ciclo precedente, e o consolida e desenvolve. Trata-se de um procedimento dinâmico, contínuo e sem fim, que coloca em questão fatos, teorias e explicações, confrontandoos com hipóteses antagonistas. 
E toda a pesquisa científica implica em uma teoria como base e um método como procedimento. A teoria, como forma de produção do pensamento humano, nos fornece um conjunto coerente de regras capazes de assegurar a explicação de um conjunto importante de fatos (Beaugrand, 1984). A teoria pode também ser considerada como uma forma de produção do pensamento humano, que cria um quadro de categorias ou conceitos, visando a explicar o fenômeno estudado (Rey, 1998).

O modelo teórico é uma representação lógica abstrata, utilizada para explicar fenômenos específicos que têm como proposta a ordenação dos seus elementos segundo uma configuração que lhe é própria (Lambotte, et al., 1995). Toda teoria, portanto, pressupõe um conjunto dinâmico, em constante modificação, cuja característica essencial é ser refutável.

O método (pressuposto básico da ciência), por outro lado, é uma lógica que supõe uma determinada concepção da realidade, de acordo com o modelo teórico. Todo método consiste essencialmente em: formular enunciados, propor hipóteses ou sistemas de enunciados e colocá-los à prova (Beaugrand, 1984); integrar idéias, métodos e técnicas que definem a especificidade de como abordar o objeto de investigação (Rey, 1996); possibilitar a construção de um conhecimento que, mesmo não sendo um reflexo puro do objeto, represente ou um momento do objeto susceptível de enriquecimento no próprio processo histórico de conhecimento, ou um momento essencial de desenvolvimento da consciência que possibilite para chegar ao objeto em um próximo nível do conhecimento (Rey, 1996). A teoria e o método são os dois pilares da pesquisa científica, que vão possibilitar a investigação do conjunto de problemas relativos ao foco de estudo.

A Psicologia, como ciência humana de grande complexidade, sofre influências histórico-culturais tanto internas (do próprio indivíduo ou indivíduos) como externas (do meio social e da cultura) que interferem na visão do pesquisador e na construção da pesquisa. Isto porque, de forma geral, a experiência consciente, imediata, empírica e, pode-se até dizer, sensorial do pesquisador, está envolvida na investigação, o que implica em importantes 
dificuldades no controle das variáveis, tornando a pesquisa científica complexa.

Em psicologia clínica tal complexidade fica ainda mais evidente quando é adotado o modelo qualitativo de pesquisa científica, pois este implica em um processo personalizado e dinâmico de investigação. Trata-se de um procedimento essencialmente construtivo-interpretativo, que tem suas raízes históricas na Antropologia Cultural, e pode incluir: a integração de fragmentos de um processo em uma nova configuração; a integração da informação obtida na investigação a partir de um conjunto de aspectos qualitativos; a interpretação como o elemento de contato na investigação da realidade estudada; ou ainda a transferência como o instrumento da investigação.

Assim, a metodologia qualitativa de pesquisa em psicologia clínica considera a ciência como uma construção da subjetividade humana, em uma forma particular e dentro de um determinado sistema teórico. Considera também que essa forma de fazer ciência apresenta uma epistemologia específica, na qual a investigação é construída dentro do fenômeno estudado. É neste sentido que os princípios teóricos dessa metodologia de pesquisa legitimam o conhecimento por construção.

A epistemologia específica dessa maneira de investigar parte do princípio que a própria investigação interfere no objeto a ser investigado e, por isso, não há neutralidade possível. A pesquisa está sempre associada à realidade e à subjetividade do pesquisador. Este é o que Rey (1998) denominou como princípio do indeterminismo: o pesquisador atua sobre o aquilo que pretende estudar, a partir de uma interação.

Portanto, há influências diretas e indiretas, conscientes e inconscientes do pesquisador na própria ciência que produz, tendo em vista que a sua produção sofre interferências de sua história e de seu funcionamento psicológico. Assim, considera-se que a pesquisa qualitativa em psicologia clínica realiza uma ciência de viabilidade, pois não pretende uma verificação direta dos resultados e conclusões mas visa explicar, apontar para um sentido da realidade, do fenômeno ou do processo estudado. 
O pesquisador é, então, um construtor de informações e os instrumentos (testes, entrevistas, questionários etc...) ou o processo que utiliza (psicoterapia, psicanálise etc...), são indutores da interação, isto é, formas de estabelecer um certo tipo de interação que pode permitir ao pesquisador estar simultâneamente em diversas posições: cientista, analista, sujeito, observador.

Portanto, pode-se afirmar que a pesquisa qualitativa em psicologia clínica é sempre uma pesquisa-ação, pois conforme a ação vai sendo construída, ela é também investigada e interpretada, e com isso, o próprio processo vai sendo modificado. Desta forma, a construção ocorre simultâneamente à produção de informações e, para tanto, necessita categorias teóricas que sejam processuais e dinâmicas.

Nesse processo de pesquisa, o pesquisador deve delinear um problema que, em geral, é bem complexo, e na própria investigação é que irá delineando os limites e especificando os indicadores que legitimam as categorias propostas. Assim, os indicadores derivam das técnicas empregadas e são construídos no caso individual.

Essa metodologia de investigação visa principalmente a detectar as regularidades contidas em um conjunto de elementos que, direta ou indiretamente, poderão adquirir uma significação para a teoria, seja porque ainda não foram integradas na construção teórica ou vêm a confirmar hipóteses teóricas específicas. Assim, essa forma de pesquisa científica busca realizar uma integração teórica sobre as relações entre eventos ou processos. Alguns sentidos de tais relações podem ser particularidades que só fazem sentido dentro do processo específico.

Apresento, a seguir, um diagrama ilustrativo do ciclo de pesquisa qualitativa em psicologia clínica, sub-dividido em suas etapas principais: enunciado do problema; plano de pesquisa, produção das observações/interpretações; análise e interpretação dos dados ou do processo; reformulação do modelo ou da teoria e publicação. 


\section{Ciclo da Pesquisa Qualitativa em Psicologia Clínica}

\begin{tabular}{|l|}
\hline Enunciado do Problema \\
\hline - Teoria \\
\hline • Observação \\
\hline$\bullet$ Levantamento da literatura \\
\hline - Escolha dos sujeitos \\
\hline Hipóteses teóricas \\
\hline
\end{tabular}

\section{$\Downarrow \pi$}

\begin{tabular}{|c|}
\hline Plano de Pesquisa \\
\hline - Escolha de um método \\
\hline - Verificação da pertinência do \\
método e das técnicas emprega- \\
das \\
\hline $\begin{array}{c}\text { Tradução das hipóteses teóricas em } \\
\text { suas implicações empíricas ou proces- } \\
\text { suais }\end{array}$ \\
\hline
\end{tabular}

$\Downarrow \Uparrow$

\begin{tabular}{|l|}
\hline $\begin{array}{c}\text { Produção das observa- } \\
\text { ções/interpretações }\end{array}$ \\
\hline • Construção da ação \\
• Registro \\
\hline • Interpretação \\
\hline Observação/Participação \\
\hline
\end{tabular}

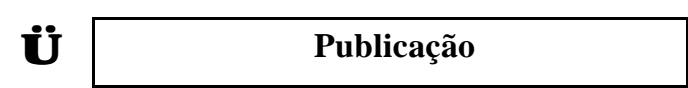

\section{$\Downarrow \Uparrow$}

$\Leftrightarrow$\begin{tabular}{|c|}
\hline $\begin{array}{l}\text { Reformulação do modelo ou da teoria } \\
\text { neralizações ou leis }\end{array}$ \\
\hline • Acomodação ou refutação \\
\hline - Novas questões \\
\hline Novas Hipóteses \\
\hline
\end{tabular}

\section{$\Downarrow \Uparrow$}

\begin{tabular}{|c|}
\hline $\begin{array}{c}\text { Análise e interpretação } \\
\text { dos dados ou do processo }\end{array}$ \\
\hline$\bullet$ Tradução das observações em dados \\
\hline • Descrição \\
\hline Construção das hipóteses \\
\hline $\begin{array}{c}\text { Reconhecimento das generalizações } \\
\text { empíricas e das leis }\end{array}$ \\
\hline
\end{tabular}


Diversas teorias psicológicas têm sido usadas como base na pesquisa qualitativa em Psicologia Clínica, sendo a Psicanálise a mais fecunda dentre elas. No entanto, é importante esclarecer que a Psicanálise difere de outros modelos teóricos de investigação na área, pois também propõe um modelo específico de pesquisa. Assim, a Psicanálise é um método de pesquisa e um método de tratamento (Baptista, 2000) do qual deriva uma técnica de psicoterapia.

Nos últimos anos muito tem sido produzido em pesquisas qualitativas baseadas na Psicanálise, seja para o estudo de obra de psicanalistas ou outros autores, projetos de psicanálise aplicada à clínica, estudos de casos, estudo de procesos psicoterapêuticos etc., mas também em pesquisa psicanalítica propriamente dita.

Na pós-graduação em Psicologia Clínica do Instituto de Psicologia da USP, a primeira Dissertação de Mestrado em Psicanálise foi a de Yutaka Kubo, Algumas Considerações sobre a Inveja do Pênis: Breve Estudo de um Caso Clínico, em 1969, sob a orientação do Prof. Dr. Durval Marcondes, psicanalista e fundador da Clínica Psicológica do mesmo Instituto. A segunda Dissertação de Mestrado em Psicanálise apresentada no IPUSP ocorreu em 1983, sob a orientação do Prof. Dr. Luiz Carlos Nogueira, psicanalista de orientação lacaniana também do Departamento de Psicologia Clínica (Batista Pinto \& Vaisberg, 2001).

A partir dessa década, o interesse tanto pela pesquisa com a Psicanálise como pela pesquisa em Psicanálise foi aumentando progressivamente, resultando na criação de uma linha de pesquisa específica em Psicanálise, na área de concentração Psicologia Clínica da Pós-Graduação do IPUSP.

Dentre os psicanalistas pioneiros nos estudos da Psicanálise em Lacan no Brasil, o professor Luiz Carlos Nogueira foi certamente um dos mais produtivos. Professor Associado do Departamento de Psicologia Clínica do Instituto de Psicologia da Universidade de São Paulo, defendeu seu doutorado em 1973 e apresentou sua tese de Livre-Docência em 1998. Seu interesse nos últimos anos foi a formalização da Psicanálise, levando em conta a linguagem como é tratada nesse campo, como cadeias de significantes que 
indicam o sujeito do inconsciente no processo de uma relação discursiva, tendo por base a obra de Jacques Lacan e suas articulações com a lógica simbólica, principalmente a Teoria dos Conjuntos.

Conheci Luiz Carlos, no início dos anos 70, como professor e supervisor de psicoterapia no curso de graduação em psicologia do IPUSP. Já naquela ocasião ele estudava Lacan, e se interessou pela minha contribuição em sala de aula a respeito dos conceitos de significado/significante em Saussure.

Muitos anos depois, voltei à sua convivência como colega no Departamento de Psicologia Clínica do IPUSP. Foi nesta condição que fui uma das responsáveis - juntamente com a Profa. Associada Jussara Brauer e Profa. Dra. Sonia Meyer - pela disciplina Metodologia Científica em Psicologia Clínica no programa de Pós-Graduação em Psicologia Clínica, no ano de 1999. Nessa oportunidade, convidei o Luiz Carlos para apresentar e discutir com os alunos suas idéias sobre a pesquisa em psicanálise. Em função do profundo conhecimento do convidado e do meu interesse especial pelo assunto, filmei em vídeo a sua palestra, ocorrida no dia 6 de Outubro de 1999.

É o registro da palestra do Professor Associado Luiz Carlos Nogueira que será apresentado a seguir, como uma homenagem póstuma ao querido professor e colega que significativas contribuições fez para a nossa ciência.

Batista Pinto, E. (2004). The qualitative research in Clinical Psychology. Psicologia USP, 15(1/2), 71-80.

\begin{abstract}
This article discusses the scientific research qualitative methodology in clinical psychology, considering science as a human subjectivity construct, and searching to understand its particularities. This methodology implies a dynamic process of investigation in a certain theoretical system. So, the qualitative research is always considered a research-action because the action is investigated and interpreted as it is being performed, modifying its own process.
\end{abstract}

Index terms: Methodology. Qualitative research. Clinical psychology. Science. Psychology. 


\section{A Pesquisa Qualitativa em Psicologia Clínica e a Pesquisa em Psicanálise}

Batista Pinto, E. (2004). La recherche qualitative dans la Psychologie Clinique. Psicologia USP, 15(1/2), 71-80.

Résumé: Cet article discute la méthodologie qualitative de recherche scientifique dans la psychologie clinique, en considérant la science comme une construction de la subjectivité humaine et en cherchant une compréhension de ses particularités. Cette méthodologie implique un processus dynamique d'investigation en un système théorique spécifique. Donc, la recherche qualitative est toujours une recherche-action, car selon l'action est construit elle est aussi investigué et interprété, en modifient le propre processus.

Mots clés: Méthodologie. Recherche qualitative. Psychologie clinique. Science. Psychologie.

\section{Referências}

Baptista, M. L. A. (2000). O método psicanalítico. Revista Brasileira de Psicanálise, 34(1), 111-130.

Batista Pinto, E., \& Vaisberg, T. M. J. A. (2001). Psicanálise e Universidade: Perspectivas. Psicologia USP, 12(2), 137-145.

Beaugrand, J. P. (1984). Démarche scientifique et cycle de la recherche. In M. R. Robert et al., Fondements et étapes de la recherche scientifique en Psychologie. Québec: Edisem.

Kuhn, T.S. (1972). La structure des révolutions scientifiques. Paris: Flammarion.

Kubo, Y. (1969). Algumas considerações sobre a inveja do pênis: Breve estudo de um caso clínico. Dissertação de Mestrado, Instituto de Psicologia, Universidade de São Paulo, São Paulo.

Lambotte, M.-C. et al. (1975). La Psychologie et ses méthodes. Paris: Fallois.

Nogueira, L. C. (1972). Contribuição ao estudo crítico do inconsciente freudiano. Tese de Doutorado, Instituto de Psicologia, Universidade de São Paulo, São Paulo.

Nogueira, L. C. (1997). A psicanálise: Uma experiência original; o tempo de Lacan e a nova ciência. Tese de Livre-Docência, Instituto de Psicologia, Universidade de São Paulo, São Paulo. 


\section{Elizabeth Batista Pinto}

Rey, F. G. (1996). Problemas epistemológicos de la Psicología. La Habana: Academia.

Rey, F. G. (1998). Curso de Metodologia Científica em Psicología. Palestras realizadas no Departamento de Psicologia Clínica do Instituto de Psicologia da Universidade de São Paulo.

Recebido em 09.06.2004

Aceito em 06.08.2004 


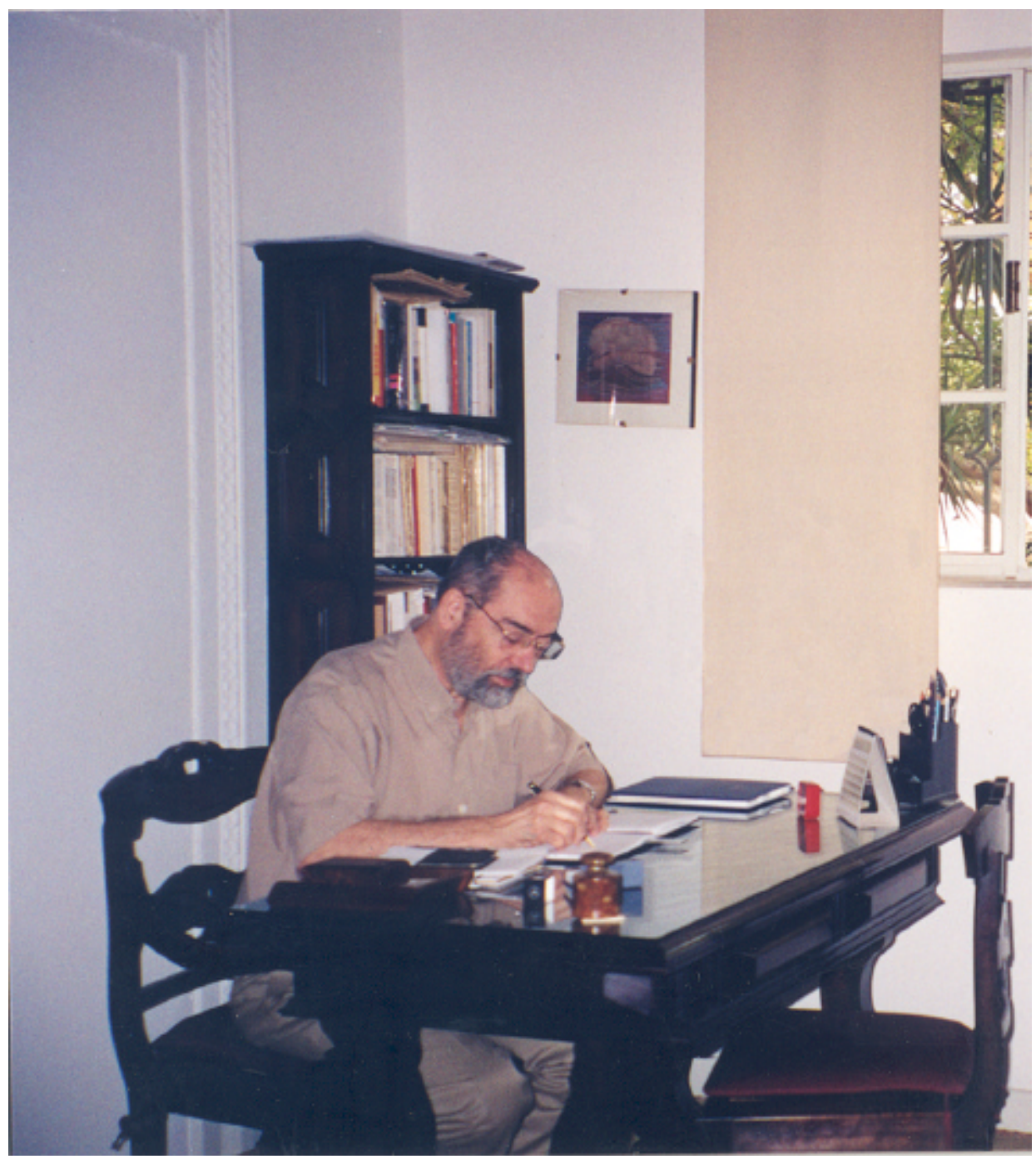

